\title{
INFLUENCIA DE LA METODOLOGÍA CONSTRUCTIVISTA SOBRE LA MOTIVACIÓN EN LA ETAPA DE EDUCACION INFANTIL
}

\author{
Ma Victoria Montesinos García \\ Universidad Complutense de Madrid \\ mvictoria.mg@hotmail.com \\ Enrique Navarro Asencio \\ Universidad Complutense de Madrid \\ enriquen@ucm.es
}

Recepción Artículo: 12 mayo 2021 Admisión Evaluación: 12 mayo 2021

Informe Evaluador 1: 17 mayo 2021

Informe Evaluador 2: 19 mayo 2021

Aprobación Publicación: 02 junio 2021

\section{RESUMEN}

La motivación tiene un peso importante en Educación Infantil. Esta es un constructo que tiene vital importancia en el desarrollo del niño. Por ello, el objetivo principal de estudio es comprobar el efecto de dos metodologías de enseñanza distintas en el grado de motivación en Educación Infantil. La metodología empleada en esta investigación se enmarca dentro de de los diseños cuasi-experimentales, de carácter cuantitativo, con un procedimiento pre-test - post-test con un grupo de control no equivalente. Se ha diseñado un instrumento adhoc que evalúa el nivel de motivación inicial de los estudiantes y se analiza la motivación al final de una intervención. El instrumento se ha aplicado a una muestra de 73 alumnos de 4 y 5 años de un colegio público de la Región de Murcia. Se estudiaron las diferencias iniciales entre los grupo y se aplicó estadística no paramétrica, calculando Ios tamaños del efecto para establecer las diferencias entre los dos grupos. Los resultados muestran que la motivación total de los alumnos que formaban las aulas experimentales y trabajaron con una metodología constructivista, aumenta considerablemente tras la aplicación de las actividades, obteniendo los valores más significativos en la motivación intrínseca. Los resultados permiten demostrar que la motivación de los alumnos es mayor cuando estos trabajan con actividades experimentales, vivenciales y manipulativas aplicadas en base a una metodología constructivista.

Palabras clave: diseño cuasi-experimental; metodología constructivista; educación infantil; motivación intrínseca; motivación extrínseca

\section{ABSTRACT}

Influence of the constructivist methodology on motivation in early childhood education. Motivation plays an important role in Early Childhood Education. As far as motivation is concerned, it is a con- 


\section{INFLUENCIA DE LA METODOLOGÍA CONSTRUCTIVISTA SOBRE LA MOTIVACIÓN EN LA ETAPA DE EDUCACIÓN INFANTIL}

struct that is of vital importance in the child's development. Therefore, the main objective of this study is to test the effect of two different teaching methodologies on the degree of motivation in Early Childhood Education. The methodology used in this research is framed within the quasi-experimental designs, of a quantitative nature, with a pre-test - post-test procedure with a non-equivalent control group. An ad hoc instrument was designed to assess the initial level of motivation of students and to analyze motivation at the end of an intervention. The instrument was applied to a sample of 73 students of 4 and 5 years of age from a public school in the Region of Murcia. The initial differences between the groups were studied and nonparametric statistics were applied, calculating the effect sizes to establish the differences between the two groups. The results show that the total motivation of the students who formed the experimental classrooms and worked with a constructivist methodology, increases considerably after the application of the activities, obtaining the most significant values in intrinsic motivation. The results show that students' motivation is higher when they work with experimental, experiential and manipulative activities applied on the basis of a constructivist methodology.

Keywords: quasi-experimental design; constructivist methodology; infant education; intrinsic motivation; extrinsic motivation

\section{INTRODUCCIÓN}

Esta investigación estudia los efectos de la metodología de enseñanza empleada en segundo ciclo de educación infantil, comparando un enfoque constructivista frente a uno tradicional, sobre la motivación para el aprendizaje. A modo de contextualización se describe la evolución de esta etapa educativa en España, también se lleva a cabo una definición de la motivación, su importancia en el aprendizaje y cómo influye en el desarrollo de los diferentes contenidos desarrollados por la metodología de enseñanza constructivista.

Según lo establecido en el artículo 12 de la Ley Orgánica de Educación (LOE) 2/2006 (LOE, 2006) la Educación Infantil constituye una etapa educativa con entidad propia y carácter voluntario que atiende a niñas y niños desde el nacimiento hasta los seis años con la finalidad de contribuir a su desarrollo físico, afectivo social e intelectual. Esta definición hace referencia a la LOE en el año 2006, pero ya se empieza a considerar importante la inclusión de la etapa de Educación Infantil en las instituciones de enseñanza pública con la LOGSE (Ley Orgánica General del Sistema Educativo) del 3 de octubre de 1990 (Batres, 2016) cuya finalidad es contribuir al desarrollo físico, afectivo, social e intelectual del niño (Calvo, 1994).

Sin embargo, la etapa de Educación Infantil es una etapa no obligatoria y sólo se imparte en los centros escolares el segundo ciclo, es decir, de los tres a los seis años de edad. En este ciclo queda establecido por la LOE (2006) una serie de competencias que se deben abordar, pues en esta etapa se sientan las bases para el desarrollo personal y social y se integran aprendizajes que están en la base del posterior desarrollo de competencias que se consideran básicas para todos los niños.

Finalmente, es importante resaltar que no existe un único método ni metodología concreta que responda a una intencionalidad educativa, sino que el/la docente debe adecuarse a las características de su alumnado a través de una atención individualizada, teniendo en cuenta también los recursos personales y materiales de los que disponga en centro donde se va a poner en marcha el proceso de Enseñanza-Aprendizaje. Por este motivo, Hernández y González (2015) destacan la necesidad de desarrollar metodologías que fomenten un aprendizaje que lleve a los discentes a desarrollar conocimientos, actitudes y estrategias que le permitan enfrentarse al mundo académico, al entorno social y posteriormente a la vida laboral.

Qué es la motivación, y su importancia en el proceso de Enseñanza-Aprendizaje De acuerdo con Pérez-Solís (2003):

La motivación se concibe como un proceso psicológico que desde el interior impulsa a la acción y determina la realización de actitudes y tareas educativas, que contribuye a que el alumno participe en ellas de forma activa y persistente, posibilitando el aprendizaje y la adquisición de conocimientos y destrezas y el desarrollo de la competencia (citado en Hernández y González, 2015, p.5). 
En este sentido, es necesario conocer por qué o por quién viene determinado dicho proceso que impulsa a la acción. Diferenciamos entonces entre motivación intrínseca y motivación extrínseca. Según Sanz (2015), la motivación intrínseca plantea que gran parte de la actividad humana para implicarse en una actividad, se realiza por su propio valor o por el interés que su ejecución conlleva, encontrando la recompensa en la realización de propia tarea (citado en Blanco, 2017). Esta motivación se produce en ausencia de estímulos externos que hagan que se modifique. Sin embargo, la motivación extrínseca hace referencia a los factores del ambiente que nos llevan a dar una determinada respuesta, a repetirla y, finalmente, a adquirirla. Esta surge a partir del valor de los incentivos que tengan los estímulos para el sujeto. Estos estímulos hacen referencias a recompensas externas, que pueden ser materiales o sociales (Sanz et al., 2015).

Según Hernández y González (2015, p. 3), se debe tener en cuenta que:

La enseñanza es una de las actividades más complejas en nuestra sociedad, ya que trabaja con un recurso tan deslumbrante como el intelecto humano e implica asistir, acompañar y estimular el saber, el hacer, el aprender y el ser. Por tal razón, la motivación en el aula ordena y dirige el comportamiento convirtiéndose así en una matriz importante del proceso de Enseñanza-Aprendizaje. El alumno necesita una fuerte motivación para aprender y desarrollar conocimientos, actitudes y estrategias que le permitan enfrentarse al mundo académico, al entorno social y posteriormente a la vida laboral.

De acuerdo con esto, es necesario conocer y comprender el desarrollo del niño (entendido desde el enfoque constructivista de Piaget como un ente activo que construye sus propios conocimientos) para ver qué factores influyen en su aprendizaje. Hablamos entonces de lo que se conoce como la motivación del logro. Autores como Verodd (1969) y Stipek (1984) han puesto de manifiesto la existencia de cambios evolutivos en las características de la motivación en las tareas escolares. Los niños hasta los 7-8 años están más orientados hacia la resolución de la tarea y no les preocupa la motivación externa. Sin embargo, a partir de esta edad, suele decrecer el interés por los aprendizajes. Por lo tanto, los determinantes de las actividades y tareas escolares son, primero intrínsecos y luego extrínsecos (López y Ruiz, 2010).＜noBreak>：Ｓｅgún López y Ruiz (2010), la motivación tiene un peso importante durante la Educación. Durante la infancia, los alumnos se encuentran en una fase de curiosidad y apego. En lo que se refiere a la motivación, en la edad de infantil esta es un constructo que tiene vital importancia a la hora del desarrollo del niño a todos los niveles. Por este motivo, se debe aprovechar esa curiosidad como fuente de motivación para aprender y desarrollar nuevos conocimientos. Deci (1976) defiende que, en esta edad, los niveles de motivación de los alumnos son muy altos, siendo la motivación intrínseca la que mayor peso tiene (citado en Blanco, 2017). Esto ocurre por esa curiosidad y el gran interés que tienen los alumnos por el conocimiento del mundo, que posteriormente, desciende en conseguir las cosas a partir de recompensas externas y no por el puro placer de aprender. Por este motivo, es importante trabajar y mantener la motivación del alumnado, para que esto no ocurra. De este modo, estamos intentando prevenir que no cambie el tipo de motivación predominante durante la infancia, estableciendo ciertas premisas que se puedan seguir cumpliendo con el paso de los años, lo cual favorecerá el aprendizaje del niño (Blanco, 2017).

La metodología constructivista como fuente de motivación en Educación Infantil En lo que se refiere a la motivación, en la edad de cero a seis años, esta es un constructo que tiene vital importancia a la hora del desarrollo del niño en todos los niveles. Por este motivo, se debe aprovechar esa curiosidad como fuente de motivación para aprender y desarrollar nuevos conocimientos, aplicando metodologías que, como Coll (1990) afirma, proporcionen un aprendizaje mucho más significativo, pues son las que promueven el compromiso activo del alumno, la motivación, la participación en el grupo, la interacción y las conexiones con el mundo real (citado en Muñoz, 2015).

Según Ceballos (2004), la metodología tradicional se define como el proceso de enseñanza disciplinaria en el que predominan tres características principales: en primer lugar, se considera al docente la clave del éxito de la educación, puesto que es quien organiza y elabora la materia que los alumnos aprende (magistrocentrismo); en segundo lugar, predomina el enciclopedismo, es decir, todo está programado y organizado con antelación y el 


\section{INFLUENCIA DE LA METODOLOGÍA CONSTRUCTIVISTA SOBRE LA MOTIVACIÓN EN LA ETAPA DE EDUCACIÓN INFANTIL}

manual escolar (el libro de texto) es el único documento que el alumno debe consultar; y por último, predomina el verbalismo y la pasividad, es decir, se utiliza el mismo modelo de enseñanza (citado en Campos, 2014, p.9).

La Teoría Constructivista de Aprendizaje defiende que el conocimiento es único en cada persona, en su propia reconstrucción interna y subjetiva de la realidad. Así, este modelo de enseñanza se basa en promover la participación activa del alumno en su proceso de enseñanza-aprendizaje (Coll, 1990). Asumiendo lo reflejado anteriormente, la educación debe tender hacia un modelo constructivista, en el que el aprendizaje sea lo más significativo, es decir, un aprendizaje que pretende partir de lo que los niños/as ya saben, esquemas, hipótesis...etc., ante la temática que se debe abordar (Leiva, 2005) y aplicado posible, y esto se consigue favoreciendo metodologías experienciales, vivenciales, manipulativas y lúdicas, que generen en los alumnos las ganas y motivación por el aprendizaje. Incrementando esa motivación en el proceso de enseñanza-aprendizaje los conocimientos, procedimientos y actitudes que se asienten en el niño lo harán de forma más duradera, lo que le permitirá desarrollar esa conciencia y empatía global de las que la sociedad y el planeta están tan necesitados (Serrano y Pons, 2011).

\section{OBJETIVOS DE LA INVESTIGACIÓN}

Esta investigación se centra en demostrar cómo influye sobre la motivación de los alumnos el desarrollo de una metodología de carácter experiencial y manipulativa en la etapa de Educación Infantil.

Por tanto, el objetivo general es comprobar si la metodología constructivista tiene una mayor incidencia en la motivación de los niños durante el desarrollo del segundo ciclo en la etapa de Educación Infantil, frente a la metodología tradicional.

\section{MUESTRA Y/O PARTICIPANTES}

El centro educativo sobre el que se emprende la indagación empírica, se encuentra ubicado en una zona deprimida de Cartagena (Los Mateos)- Murcia y con necesidades de compensación educativa, y alberga a familias en riesgo de exclusión social.

En este estudio participaron cuatro aulas compuestas por alumnos de cuatro y cinco años. La muestra la formaron un total de 73 alumnos (39 alumnos en las aulas control y 34 en las aulas experimentales). Es interesante señalar que los ninguno de estos alumnos han sido diagnosticados con Necesidades Educativas Especiales (NEE).

\section{METODOLOGÍA Y/O INSTRUMENTOS UTILIZADOS}

Esta investigación se enmarca dentro de los diseños cuasi-experimentales, siendo un estudio con la aplicación pre-test y pos-test con grupo de control no equivalente. Para llevar a cabo esta investigación se ha diseñado y aplicado una intervención educativa compuesta por actividades en las que se abordaron contenidos relacionadas con una de las áreas que abarca el currículum del segundo ciclo de la etapa de Educación Infantil, en concreto basadas en el Decreto 254/2008 de la Región de Murcia.

El proceso que se ha seguido ha sido el siguiente:

En un primer momento, las cuatro aulas del centro donde se ha realizado la intervención han sido seleccionadas de forma incidental, dos aulas con alumnos de 4 años y dos aulas con alumnos de 5 años. El tipo de metodología a aplicar se otorgó de forma aleatoria. Para ello, se convocó previamente a los docentes tutores y se les explicó cómo debían llevarse a cabo el proyecto de actividades, facilitándoles también todos los materiales necesarios para ello.

En las aulas A-Control se aplicó una metodología tradicional, que consistió en impartir los contenidos seleccionados a través de una explicación previa para la cual se podría utilizar la pizarra tradicional, póster de los contenidos (Ios números, las vocales, los colores, etc.), y se trabajará con fichas y materiales tradicionales (ceras de colores o lápices), y de forma individual, aunque los alumnos podían estar sentados en grupos de mesa. Las acti- 
vidades de estas intervenciones duraron menos de una hora cada una de ellas, pues eran fichas de trabajo donde los alumnos debían reflejar los conocimientos adquiridos durante la explicación del docente.

Para conocer el grado de motivación se utilizó un instrumento de observación elaborado ad hoc, concretamente una escala de estimación, ya que las pruebas existentes se dirigen a edades superiores, por ejemplo el test AMPET de motivación propuesto por Ruiz, Graupera, Gutiérrez y Nishida, (2004) para edades comprendidas entre nueve y doce años, o la escala CEAP-48 que evalúa la motivación de alumnos de secundaria (Barca, Porto, Santorum, Brenlla, Morán y Barca, 2005).

Los indicadores elaborados se sometieron a validación por parte de 8 expertos (docentes de Educación Infantil, Doctores en psicología educativa y profesionales de metodología) para determinar la calidad de la redacción, su pertinencia y relevancia. Se calcularon índices de acuerdo (W de kendall) y se hicieron las modificaciones propuestas, añadiendo explicaciones y ejemplo a algunos de los ítems y ítems y descartando los indicadores de la dimensión vinculada a la relación con familia.

El instrumento está compuesto por ítems relacionados con las dos grandes dimensiones de la motivación académica (intrínsecos y extrínsecos) tal y como se justifica en el marco teórico.

\section{RESULTADOS ALCANZADOS}

En cuanto a los resultados obtenidos en las variables de motivación en los grupos (control y experimental) antes de comenzar la intervención, es decir, los resultados obtenidos en la prueba pre-test. En la Tabla 1 se puede observar que existen diferencias iniciales estadísticamente significativas en motivación intrínseca ( $p$ valor $=0,00$ con un tamaño del efecto grande $=0,538$ ), extrínseca ( $p$ valor $=0,009$ con un tamaño del efecto medio $=0,308$ ), y total ( $p$ valor $=0,000$ con un tamaño del efecto grande $=0,567$ ), todas ellas a favor del grupo experimental ( $p$ valor $=0,337$ ). Por tanto, el grupo experimental parte de un mayor grado de motivación antes de comenzar la intervención.

Tabla I. Comparación en los niveles iniciales de motivación

\begin{tabular}{|l|l|l|l|l|}
\hline \multicolumn{2}{|l|}{ GRUPO } & $\mathbf{N}$ & $\mathbf{p}$ & $\mathbf{r}$ \\
\hline $\begin{array}{l}\text { INTRINSECA_ } \\
\text { PRE }\end{array}$ & EXPERIMENTAL & 34 & 0,000 & 0,538 \\
\cline { 2 - 5 } & CONTROL & 39 & & \\
\hline $\begin{array}{l}\text { EXTRINSECA_ } \\
\text { PRE }\end{array}$ & EXPERIMENTAL & 34 & 0,009 & 0,308 \\
\cline { 2 - 5 } & CONTROL & 39 & & \\
\hline \multirow{2}{*}{ SOCIAL_PRE } & EXPERIMENTAL & 34,00 & 0,377 & 0,104 \\
\cline { 2 - 5 } & CONTROL & 39 & & \\
\hline $\begin{array}{l}\text { MOTIVACION } \\
\text { _TOTAL_PRE }\end{array}$ & EXPERIMENTAL & 34 & 0,000 & 0,567 \\
\cline { 2 - 5 } & CONTROL & 39 & & \\
\hline
\end{tabular}

En cuanto a los resultados obtenidos en la variable motivación en los grupos (control y experimental) después de llevar a cabo la intervención, es decir, los resultados obtenidos en la prueba post-test. Se observa en el gráfico 2 que existen diferencias estadísticamente significativas en motivación intrínseca ( $p$ valor $=0,000$ con un tamaño del efecto muy grande $=0,869$ ) y extrínseca ( $p$ valor $=0,000$ con un tamaño del efecto grande $=0,698$ ). Por tanto este grupo, aunque partía con un mayor grado en todas las dimensiones de motivación con respecto al grupo control, después de la intervención el tamaño de esas diferencias ha aumentando en más de un $30 \%$.en la motivación intrínseca y total. 
Tabla II. Comparación en los niveles finales de motivación

\begin{tabular}{|l|l|l|l|l|}
\hline \multicolumn{2}{|l|}{ GRUPO } & N & p & r \\
\hline INTRINSECA_POST & EXPERIMENTAL & 34 & 0,000 & 0,869 \\
\cline { 2 - 5 } & CONTROL & 39 & & \\
\hline \multirow{2}{*}{ EXTRINSECA_POST } & EXPERIMENTAL & 34 & 0,000 & 0,698 \\
\cline { 2 - 5 } & CONTROL & 39 & & \\
\hline \multirow{3}{*}{ SOCIAL_POST } & EXPERIMENTAL & 34,00 & 0,000 & 0,578 \\
\cline { 2 - 5 } & CONTROL & 39 & & \\
\hline $\begin{array}{l}\text { MOTIVACION_TOTA } \\
\text { L_POST }\end{array}$ & EXPERIMENTAL & 34 & 0,000 & 0,861 \\
\cline { 2 - 5 } & CONTROL & 39 & & \\
\hline
\end{tabular}

\section{DISCUSIÓN}

Los cambios de motivación antes y después de la intervención, de forma diferencias para los grupos de control y experimental. Primero, se observa que existen cambios estadísticamente significativos en la motivación de Ios sujetos del grupo experimental, aumentando positivamente un 91,18\% de los casos en intrínseca. Sin embargo, en el $91,18 \%$ de los casos ha disminuido su nivel de motivación extrínseca. Por tanto, a nivel general, existe una mejora en la motivación que han trabajado con una metodología constructivista. Por lo tanto, tras la intervención el 76,47\% del grupo experimental ha mejorado su motivación total, lo que ha supuesto un cambio estadísticamente significativo. En cambio, en el grupo control ha mejorado el 33,33\% de los sujetos y ha empeorado el $41 \%$, sin que suponga un cambio significativo.

\section{CONCLUSIONES}

Llegados a este punto de la investigación se concluye que la motivación es mayor cuando los alumnos trabajan con actividades experimentales, vivenciales y manipulativas aplicadas en base a una metodología constructivista, donde el niño es el principal protagonista del propio proceso de E-A. Por lo tanto, como afirma Freire (2011) la motivación del niño en el proceso de E-A aumenta trabajando de forma experimental y manipulativa en la naturaleza, al mismo tiempo que potencia en los alumnos un aprendizaje constructivista.

El hecho de trabajar de forma directa materiales de la naturaleza (vivos e inertes), ha desarrollado en los alumnos un sentimiento de empatía que ha promovido en los alumnos desarrollar hábitos de respeto y cuidado hacia el medio natural. Por tanto, se llega a la conclusión de que las escuelas necesitan desarrollar un nuevo plan de estudio que fomente el aprendizaje de los diferentes contenidos a través trabajo experimental y manipulativo, promoviendo así que estos sean significativos y perduren en el tiempo.

En síntesis, el trabajo realizado aporta información útil para reconsiderar posibles medidas orientadas a la mejora de la motivación en los alumnos de Educación Infantil a través de la metodología constructivista, pues no se debe olvidar que la motivación facilita aprendizaje (Tapia, 1997).

\section{REFERENCIAS BIBLIOGRÁFICAS}

Barca, A., Porto, A., Santorum, R. y Barca, E. (2005). Motivación académica, orientación a metas y estilos atribucionales: la escala CEAP -48. Revista de Psicología y Educación, 1, (2), 103-136. Recuperado de: http://www.revistadepsicologiayeducacion.es/pdf/18.pdf 
Blanco. F. J. (2017). Evaluación de la Motivación Académica en Niños de Primer Ciclo de Educación Infantil. (Tesis doctoral). Universidad de León, León.

Boza, A. y Toscano, M. (2012). Motivos, actitudes y estrategias de aprendizaje: aprendizaje motivado en alumnos universitarios. Revista de currículum y formación del profesorado, 16, (1), 126-140.

Calvo, M. (1994). La Educación Infantil en España. Planteamientos legales y problemática actual. (Tesis doctoral). Universidad Complutense de Madrid, Madrid. Recuperado de: https://eprints.ucm.es/3791/1/T19893.pdf

Coll, C. (1990). Un marco de referencia psicológico para la educación escolar: la concepción constructivista del aprendizaje y de la enseñanza. En Coll, C., Palacios, J. Y Marchesi, A. (eds.): Desarrollo psicológico y educación II. Psicología de la Educación. Madrid: Alianza Editorial.

Hernández, M. L., y González, M. A. (2015). La motivación en el aula: estrategia esencial para mejorar el aprendizaje en la escuela primaria. Cuadernos de Educación y Desarrollo, (55), 1-10. Recuperado de: http://atlante.eumed.net/motivacion-aula/

Kerlinguer, F. (2001). Investigación del comportamiento. MacGraw-Hill Interamericana: Distrito Federal.

López, 0. y Ruiz, C. (2010). Psicología de la Educación para maestros de Educación Primaria. Murcia: Diego Marín.

McClelland, D. (1989). Estudio de la motivación humana. Madrid: NARCEA.

Sanz, M.T., Menéndez, F.J., Rivero, M.P. y Conde, M. (2015). Psicología de la motivación: teoría y práctica. Madrid: Editorial Sanz y Torres. Recuperado de: https://www.scribd.com/doc/182928838/Psicologia-de-laMotivacion

Sanchidrian, M.C. (1991). Historia de la Educación Infantil. Redined (revista interuniversitaria), 10, (9-14). Recuperado de: http://campus.usal.es/ revistas_trabajo/index.php/02120267/article/viewFile/6909/6890

Serrano, J.M y Pons, R.M (2011). El constructivismo hoy: enfoques constructivistas en Educación. REDIE (Revista electrónica de investigación educativa), 13, (1), 1-27. Recuperado de: http://www.scielo.org.mx/scielo.php?script=sci_arttext\&pid=S160740412011000100001\&lng=es\&tlng=es.

Tapia, A. (1997). Motivar para el aprendizaje. Teoría y estrategias. Madrid, España: EDEBÉ. 
